\title{
Is fungal disease in Sri Lanka underestimated? A comparison of reported fungal infections with estimated disease burden using global data
}

\author{
PI Jayasekera $^{1,2}$, DW Denning ${ }^{2}$, PD Perera ${ }^{1}$, A Fernando $^{3}$, S Kudavidanage $^{1}$ \\ Sri Lankan Journal of Infectious Diseases 2015 Vol.5 (2):73-85 \\ DOI : http://dx.doi.org/10.4038/sljid.v5i2.8055
}

\begin{abstract}
Introduction

With an increase in the elderly population and developments in medical care, increased numbers of patients with fungal diseases are expected. This necessitates a new vision for fungal diseases in Sri Lanka, a tropical country with a population of 20 million. The objective of this study was to estimate national fungal infection caseloads using global and national epidemiological datasets.
\end{abstract}

\section{Methods}

We searched national data and surveillance studies published by us and other authors for relevant disease terms. Locally collected incidence data were available for candidaemia, fungal keratitis, cryptococcosis, allergic broncho-pulmonary aspergillosis (ABPA) and severe asthma with fungal sensitization (SAFS). Global disease estimation was done using international data. Generally, disease estimates were conservative as they assumed the lowest incidence rates reported in the literature and focused only on well-defined risk populations.

\section{Results}

Among the 1,317 HIV/AIDS patients (in 2010), there were 639 estimated oral candidiasis cases, 100 oesophageal candidiasis cases and only 13 cases of cryptococcal meningitis. The estimated prevalence of chronic pulmonary aspergillosis (CPA) post TB is estimated to be 1,443 and all forms of chronic pulmonary aspergillosis (including aspergilloma) 2,886 patients. We estimated 229 cases of invasive aspergillosis and 41 cases of mucormycosis. The projected annual incidence of candidaemia is 507 and an estimated 76 candida peritonitis cases post-surgery.

According to our estimates 10,344 patients were suffering from ABPA and 13,654 were suffering from SAFS. The projected annual incidence of fungal keratitis would be 1,277 and tinea infections would affect 50 children. Pneumocystis incidence could not be estimated.

\footnotetext{
${ }^{1}$ Department of Mycology, Medical Research Institute, Colombo

${ }^{2}$ Education and Research Centre, University Hospital of South Manchester, Manchester, UK

${ }^{3}$ Nawaloka Hospital, Colombo

${ }^{4}$ Central Chest Clinic, Colombo
}

Address for correspondence: Dr. Primali I. Jayasekera, Department of Mycology, Medical Research Institute, Colombo, Sri Lanka. Telephone: +94112698725 e-mail: jayali_jaya@yahoo.com Received

6 February 2015 and revised version accepted 20 September 2015 


\section{Conclusion}

Our estimates suggest that candidaemia and invasive aspergillosis could be the leading causes of fungal-associated deaths in Sri Lanka whereas globally it is due to SAFS, ABPA and CPA. More precise data from the whole country is needed to validate these estimates. Such data would be very useful for developing healthcare policies for prevention, diagnosis and treatment of serious fungal infections.

Keywords : Serious Fungal Infections, Disease Burden, Sri Lanka

\section{Introduction}

The number of people with fungal infections is not known for certain anywhere in the world. Some data show a rise in incidence. ${ }^{1}$ For example, national autopsy data from Japan between 1969 and 1994 showed increased prevalence of visceral mycoses. National hospital admission data in Australia between 1995 and 1999 showed increasing hospitalization for candidiasis and aspergillosis. ${ }^{1}$ Single-centre data from India also show an increase in mucormycosis over time. ${ }^{1}$ Asthma rates have increased and probably therefore the number of people with allergic aspergillosis. On the other hand, the increasing rollout of antiretroviral therapy in highly endemic areas has probably reduced Pneumocystis pneumonia, mucosal candidiasis and cryptococcal meningitis, although data are scanty. We therefore attempted an estimate of fungal infection incidence and prevalence in Sri Lanka.

Sri Lanka is a tropical island (with a land area of $65,525 \mathrm{Sq} . \mathrm{km}^{2}$ ) in the Indian Ocean with a population of about 20 million. $^{2}$ Sri Lanka had graduated from a low to middle income country (GDP US\$ 4300) $)^{3}$ and the GDP growth rate was $8.5 \%$ in $2010 .^{4}$ The population pyramid has changed with an increase in the elderly population ${ }^{5}$ defined as chronological age of $60+$ years. $^{6}$ There is a low birth rate and an increase in life expectancy (increase of 11.1 years in males and 15.4 years in females over 50 years). ${ }^{3}$ Liver and renal transplantations have been initiated in a few centers in recent years. The transplanted population is therefore steadily increasing. In addition, increasing numbers of oncology units and early detection of cancers has increased the number of immunocompromised patients (unpublished data). Sri Lanka has very few HIV/AIDS patients but the numbers are rising. More and more devices are used for treatment as well as usage of more broad-spectrum antibiotics predisposing patients to fungal diseases such as mucosal and systemic candida infections. These demographic and healthcare shifts presage a larger fungal caseload over the coming years.

In Sri Lanka, healthcare is provided by government and private health care institutions. Government healthcare is free of charge while private healthcare has to be paid for by the patient. The government healthcare system comprises the National Hospital, Colombo, Teaching hospitals based close to the 8 Faculties of Medicine, General hospitals, District hospitals and Peripheral Units with total bed strength of $1,029,914 .^{7}$ This includes 8 ICU beds per 1000 hospital beds. ${ }^{8}$ Private sector hospitals function as groups of companies with many branches in the major cities. In addition to these private hospitals, patients can just walk-in to see a consultant or a GP in the private sector. This makes data gathering very difficult as doctors and hospitals in the private sector do not code diagnoses for their patients. While government sector hospitals do keep coded diagnoses, patients seek care in more than one 
facility, leading to possible duplication of data. Only a few campaigns, including the antileprosy campaign, anti-TB campaign and National STD/AIDS Control Programme in Sri Lanka are geared to eliminate specific diseases from the island and collect and manage their data accurately. No systematic record keeping is in place for any fungal disease. We therefore used a combination of published local literature and modeling to estimate the incidence and prevalence of serious fungal diseases in Sri Lanka.

\section{Methodology}

We searched national data available from the Sri Lankan Department of Census \& Statistics, Ministry of Health, World Health Organization (WHO) and Faculty of Medicine, Colombo, as well as surveillance studies published by us and other authors for relevant disease terms. Locally collected incidence data were available for candidaemia, fungal keratitis, cryptococcosis, ABPA, fungal sensitized asthma and mucormycosis. Generally, local disease estimates were conservative as they assumed the lowest incidence rates reported in the literature and focused only on well-defined risk populations.

\section{Candidiasis in HIV/AIDS}

Estimates were done on the basis that $20 \%$ of patients not on anti- retroviral therapy (ART) develop oesophageal candidiasis annually and $0.5 \%$ of patients on ART and $90 \%$ of patients not on ART develop oral candidiasis. ${ }^{9}$

\section{Cryptococcosis}

Annual caseload was estimated according to unpublished data from the Department of Mycology, Medical Research Institute (MRI), Sri Lanka.

\section{Tuberculosis and chronic pulmonary aspergillosis (CPA)}

We started with WHO estimates of the number of new cases of PTB and of deaths from PTB and assume that the mortality figures quoted by the WHO were for the point 12 months after the diagnosis of PTB. ${ }^{10}$ Compilers of these estimates have done an extensive literature search including more than 400 papers published since 1940. They have also interviewed other researchers who had publications, but case reports were excluded from these estimates. ${ }^{10}$

Fig. $1 a$

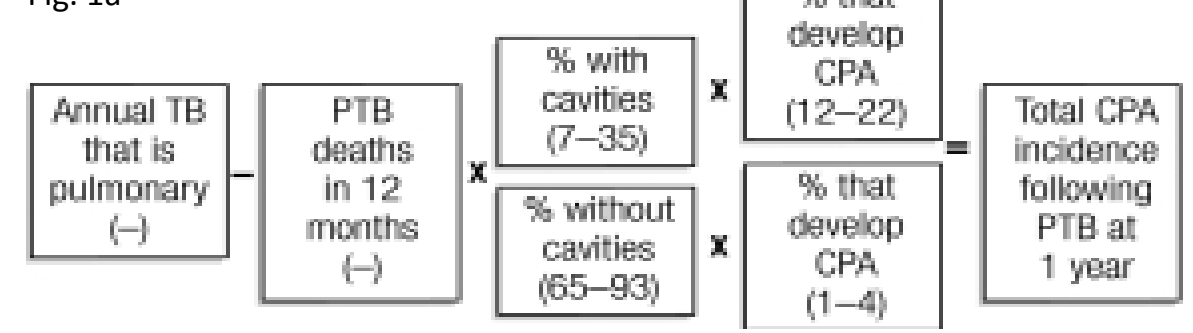

Annual no. of cases of tuberculosis of lungs with presence of cavities $(\approx 12 \%$ of total no.) $\mathrm{x}$ risk of CPA development $(22 \%)$

$\div$ no. of emerged cases of tuberculosis without cavities ( $\approx 88 \%$ of the total incidence)

$\div$ risk of $\mathrm{CPA}$ in this patients $(2 \%)$

$=$ possible cases of CPA. ${ }^{10}$

Fig. 1 Factors used to estimate the annual incidence of CPA (modified from ref 10)
As values obtained from the surveyed literature varied widely (Fig. 1a), a range rather than a single value was used to formulate the equation. 
The determination of a particular value depended on the PTB incidence of a particular country. ${ }^{10}$ Some factors were not considered in the current study as data was not available.

\section{Invasive aspergillosis (IA)}

The number of patients in this group with invasive aspergillosis was estimated using a formula suggested by Denning DW et $\mathrm{al}^{10}$ (Table 1) by addition of the numbers in each category in the group. Patients using steroids were ignored. Patients with invasive aspergillosis following other respiratory diseases were calculated separately. ${ }^{10}$

Table 1:

Calculation of number of patients in the given categories with invasive aspergillosis

\begin{tabular}{lc}
\hline & $\%$ \\
\hline AML & 10 \\
non-AML haematological conditions & 100 \\
renal transplantation & 0.5 \\
liver transplantation & 4 \\
\hline
\end{tabular}

\section{Candidaemia}

The annual candidaemia caseload was estimated using estimates of renal and liver transplants and AML incidence, (unpublished data from the Department of Mycology, MRI, Sri Lanka). ${ }^{11}$

\section{Asthma, allergic bronchopulmonary aspergillosis (ABPA) and severe asthma with fungal sensitisation (SAFS)}

Asthma affects 414,000 Sri Lankan adults (2.75\% of the adult population), and assuming ABPA prevalence is $2.5 \%$ (international mean rate), 10,344 persons are estimated of having ABPA. Thirty three percent of the worst $10 \%$ of asthmatics $(41,400)$ are estimated as having SAFS $(13,654) .{ }^{10,12}$

\section{Vulvo vaginal candidiasis}

$5-8 \%$ of all females in a given population get recurrent attacks (4 or more acute episodes annually). ${ }^{13}$

\section{Superficial fungal infections}

Based on our experience, we estimated 50 fungal hair, nail \& skin infections among Sri Lankans annually. This number could be more than that. However, definitive data is not available.

\section{Fungal Keratitis}

This number was estimated according to data from Hong Kong studies and was $6.3 / 100,000 .^{14}$ 


\section{Mucormycosis}

We estimated the annual incidence as 41 cases based on unpublished data (in 2012) from the Department of Mycology, MRI, Colombo.

\section{Rhinosporidiosis}

Rhinosporidiosis is hyper-endemic in Sri Lanka ${ }^{15}$, but no recent data is available on the caseload.

\section{Pneumocystis}

No data is available for Sri Lanka as it is diagnosed using various histological staining procedures. Hence, we could not estimate the disease burden.

\section{Results}

Table 2 summarizes the estimated annual incidence of global and Sri Lankan fungal burden.

Table 2: Annual incidence of fungal diseases

\begin{tabular}{llc}
\hline Fungal Disease & Global data $^{\mathbf{1 6}}$ & Local data \\
\hline Oral candidiasis & 10 million & 639 \\
Oesophageal candidiasis & 2 million & 100 \\
Cryptococcal meningitis & 1 million & 13 \\
Chronic pulmonary aspergillosis (post TB) & 1.2 million & 1,443 \\
Chronic pulmonary aspergillosis & $3.5-15$ million & 2,886 \\
Invasive aspergillosis & $>200,000$ & 229 \\
Candidaemia & 400,000 & 507 \\
Candida peritonitis & 100,000 & 76 \\
ABPA & 4.8 million & 10,344 \\
Severe asthma with fungal sensitisation & $3.5-15$ million & 13,654 \\
Vulvo vaginal candidiasis & 137 million & 25,750 \\
Fungal keratitis & $1-1.2$ million & 1,277 \\
Mucormycosis & Not estimated & 41 \\
Tinea infections & 985 million & 50 \\
\hline
\end{tabular}

\section{Candidiasis in HIV/AIDS}

We estimated that 100 patients with HIV/AIDS develop oesophageal candidiasis annually while 639 patients develop oral candidiasis.

\section{Cryptococcosis}

We estimated that only 3 patients with HIV/AIDS developed cryptococcal meningitis. However, the actual number of patients who develop cryptococcal meningitis following HIV/ AIDS could be greater as testing for cryptococcal antigen was only been available since 2014 in Sri Lanka and patients with AIDs could have a negative culture with a positive antigen result. According to our estimation, the annual national caseload is 13 patients, of whom 5 have no underlying disease, 3 are HIV positive and 5 either have cancer or have undergone transplantation (unpublished data from the Department of Mycology, Medical Research Institute, Sri Lanka). 
Tuberculosis and chronic pulmonary aspergillosis

According to $\mathrm{TB}$ and other respiratory death rates, we estimated the annual incidence of chronic pulmonary aspergillosis (CPA) following pulmonary TB in Sri Lanka as 1,443, assuming a $15 \%$ annual mortality. ${ }^{10}$ If we assume that TB is the underlying cause for $50 \%$ of patients with CPA, the estimated total prevalence of CPA is 2,886 , all with underlying respiratory problems.

Invasive aspergillosis (IA) complicating leukaemia, transplantation and COPD

In Sri Lanka no data is available for incidence of Acute Myeloid Leukaemia (AML) and renal transplantation. After estimating numbers for AML $(3 / 100,000)$ and renal transplants (over 500 annually), we estimated 229 cases of invasive aspergillosis, 102 in patients with respiratory diseases and 127 in immunocompromised patients.

\section{Candidaemia}

The estimated annual candidaemia caseload (in patients treated out-side ICUs) was 203 patients and for the total ICU population it was estimated as 304 patients.

\section{Table 3: Candida species distribution in blood cultures at NCISL ${ }^{17}$} and at Department of Mycology, MRI, Colombo ${ }^{11}$

\begin{tabular}{lcccc}
\hline Candida species & NCISL & NCISL & NCISL & Department of Mycology \\
& $\mathbf{2 0 0 7}$ & $\mathbf{2 0 0 8}$ & $\mathbf{2 0 0 9}$ & $\mathbf{2 0 0 1 - 2 0 1 0}$ \\
\hline C.tropicalis & $3.68 \%$ & $3.86 \%$ & $3.88 \%$ & $51.09 \%$ \\
C.glabrata & $3.88 \%$ & $2.53 \%$ & $2.42 \%$ & $33.87 \%$ \\
C.albicans & $0.19 \%$ & $0.36 \%$ & $0.60 \%$ & $10.92 \%$ \\
C.parapsilosis & $0.19 \%$ & $0.36 \%$ & $0.24 \%$ & $2.1 \%$ \\
C.guillermondii & 1 isolate & & & $0.81 \%$ \\
C.famata & & & & 1 isolate \\
C.lusitaniae & & & & 1 isolate \\
\hline
\end{tabular}

NCISL - National cancer Institute, Sri Lanka

The above data ${ }^{11,1 /}$ shows the Candida species distribution in blood cultures.

Asthma, allergic bronchopulmonary aspergillosis (ABPA) and severe asthma with fungal sensitization (SAFS)

Though asthma is a serious public health problem in Sri Lanka, there are no recent documented mortality and morbidity data for asthma, ABPA and SAFS. We anticipated 10,344 ABPA cases in Sri Lanka, but by applying a rate of $6 \%$, this number would rise to 21,600. In Sri Lanka 13,654 patients with SAFS have been estimated with the available data from other countries.

\section{Vulvo vaginal candidiasis}

No data is available on Sri Lankan patients. The estimated annual figure using global data is 25,750 .

\section{Superficial fungal infections}

Though it is very low in number, based on our experience we estimated 50 fungal hair, nail \& skin infections among Sri Lankans annually. Although the actual numbers could be much higher, data on superficial fungal infections is very scanty. 


\section{Fungal keratitis}

Our annual estimation for fungal keratitis is 1277 for Sri Lanka.

\section{Mucormycosis}

This is not an uncommon condition in patients with poorly controlled diabetes mellitus. We estimated the annual incidence as 41 cases based on unpublished data (in 2012) from the Department of Mycology, MRI, Colombo.

\section{Rhinosporidiosis}

Although reported as being hyper-endemic ${ }^{15}$, case load could not be estimated due to a lack of recent data.

\section{Pneumocystis}

No data is available for Sri Lanka as it is diagnosed by using various histological staining procedures. Hence, we could not estimate the disease burden.

\section{Estimated total fungal burden}

In Sri Lanka the estimated annual fungal disease burden is 57,009 . But the number of deaths or mortality rate couldn't be estimated as data is not available.

\section{Discussion}

The Sri Lankan population in 2012 was 20.2 million among whom $25.5 \%$ were children, $62 \%$ adults and $12 \%$ over 59 years of age. Females constitute $51.5 \%$ of the total population. ${ }^{18}$ The Sri Lankan birth and death rates in 2010 were 16.26 and 6.13 per 1000 population respectively. ${ }^{3}$ Population growth is $0.9 \%$ annually and the life expectancy at birth is 75.3 years. ${ }^{3}$

The first case of HIV was detected in 1987 in Sri Lanka. The country has a low level of HIV positive patients, with an estimated adult HIV prevalence of less than $0.1 \%$ in 2010 and an estimated 3000 adults and 35 children living with HIV. ${ }^{19}$ By the end of 2010, the reported cumulative number of HIV cases was 1,317 (784 males and 533 females) with a male-tofemale ratio of 1.5:1. Two-thirds (66.4\%) of the reported HIV cases in 2010 were among individuals aged between 30 and 49 years. ${ }^{20}$ Of the diagnosed patients, $40.6 \%$ were on antiretroviral therapy (ART) in 2009. ${ }^{9}$ There were 19 deaths reported in 2010 with a HIV seropositivity rate of $0.03 \% .{ }^{21}$ Compared to Sri Lanka, in the South East Asia region the coverage of ART is $32 \%$ among the HIV patients with advanced disease. ${ }^{22}$

Our estimate of patients with HIV/AIDS who develop oesophageal candidiasis annually was 100 while 639 patients could develop oral candidiasis. In contrast, the global burden of oesophageal candidiasis among HIV/AIDS patients is estimated as 2 million with an oral candidiasis burden of 10 million. ${ }^{16,23}$

Although the global cryptococcal meningitis burden is estimated as 1 million among HIV/AIDS patients, cryptococcal meningitis remains rare in Sri Lanka, probably because the prevalence rate of HIV is $<0.1 \% .{ }^{19}$ With the available culture results (unpublished data, 
Department of Mycology, MRI, Colombo), we estimated that only 3 patients with HIV/AIDS developed cryptococcal meningitis. However, the actual number could be more as testing for cryptococcal antigen has only been available to clinicians recently in Sri Lanka. There is no mortality data available in Sri Lanka. The estimated case fatality rate is 600,000 worldwide with a mortality of $15-20 \%$ of patients in USA and $>50 \%$ in the developing world ${ }^{23}$ It is possible that cryptococcal meningitis is under-diagnosed in immunocompromised patients in Sri Lanka. The low detection rate may be partly attributed to unawareness and lack of microbiology facilities.

Our conservative estimate of the annual national caseload of cryptococcal meningitis is therefore 13 patients, with 5 patients with no underlying disease, 3 patients with HIV and 5 patients with cancer or undergone transplantation (unpublished data from the Department of Mycology, Medical Research Institute, Sri Lanka). The caseload however could be very much larger due to the rapid increase in the immunocompromised population within Sri Lanka.

In Sri Lanka, prevalence of all forms of TB, including HIV related TB was 101 per 100,000 population in $2010,{ }^{24,25}$ with an incidence rate (including HIV) of 66 per $100,000^{25}$ and a mortality rate of 9.1 per 100,000 (excluding HIV). ${ }^{25}$ In 2010,9328 new cases of TB were identified with smear positivity in $50 \%{ }^{25}$ Among the new cases, $73 \%$ were pulmonary TB patients and $0.2 \%$ had multi drug resistant tuberculosis (MDR-TB). ${ }^{25}$ Rifampicin was used throughout the treatment period and for $90 \%$ of the patients fixed dose combinations were used. $^{25}$ Treatment success rate among the new TB smear positive cases was $86 \%{ }^{25} \mathrm{HIV}$ prevalence among new TB cases was $0.1 \%$ in $2010 .{ }^{24}$ A national policy for provision of cotrimoxazole and ART for HIV positive TB patients is in place. $^{24}$

A research group estimated that 21\% (USA) to 35\% (Taiwan, China) of pulmonary tuberculosis (PTB) patients develop pulmonary cavities and approximately $22 \%$ of these patients develop CPA. ${ }^{10}$ Further, the same researchers estimated that China and India had a five-year prevalence of 16.2 and 23.1 per 100,000 respectively for CPA following pulmonary TB. ${ }^{10}$ CPA could also account for some cases of smear-negative PTB. ${ }^{10}$

The estimated annual incidence of CPA following pulmonary TB in Sri Lanka is 1,443, and of CPA is 2,886, all with underlying respiratory problems. In contrast the total estimated global disease burden of patients with CPA is 3.5-15 million with 1.2 million following PTB and an estimated death rate of $>450,000(\sim 15 \%$ annually). Worldwide, these 2 diseases are under-diagnosed and commonly mistaken for TB. Similarly, under-diagnosis may have resulted in the low estimated incidence in the current study. ${ }^{16,23}$

As there are no data available for Sri Lanka, we estimated the number of invasive aspergillosis based on an estimated AML incidence of 3/100,000 and over 500 renal transplants annually. Using these figures, we estimated 229 cases of invasive aspergillosis annually, 102 of whom had predisposing respiratory diseases 127 in immunocompromised patients. In India, Iran and Tunisia, IA occurs in around $15 \%$ of patients with haematological malignancies. ${ }^{26}$ The incidence of IA among renal transplant patients was higher $(2-4 \%)$ in developing countries, compared to $<1 \%$ in developed countries which could be due to poor hygiene and sanitation in the former. ${ }^{26}$ IA has a $\sim 50 \%$ mortality in the developed world if 
treated but in one study from Brazil was much higher at $83 \% .^{23.26}$ The global estimate of annual incidence is $>200,000^{16}$

Candidemia is the most common form of invasive candidiasis. ${ }^{1}$ Incidence varies from 1 $12 / 1000$ admissions in India to $0.05-0.36 / 1000$ admissions in Australia. ${ }^{1}$ The estimated global disease burden is 400,000 and $>120,000$ deaths. ${ }^{16,23}$ It is estimated that it has a $40 \%$ mortality rate even if treated. ${ }^{23}$ The epidemiology of candidemia varies among countries in the Asia-Pacific region. Based on the renal and liver transplants and the estimated AML incidence, the annual candidaemia caseload was estimated as 203 patients (among patients who were not treated at ICUs). In the total ICU population it was estimated as 304 patients. ${ }^{11}$ It was estimated that 76 ICU patients get candida peritonitis annually in Sri Lanka. ${ }^{11,27}$ Knowledge of the epidemiology of Candida species may be of use in making treatment decisions before the culture results are available. ${ }^{1}$

Asthma is a significant public health problem in Sri Lanka. In 2007, 142,125 adults were hospitalized in Sri Lanka due to asthma. ${ }^{28}$ In 2010, 2,543 patients died of asthma $(1.72 \%$ of all deaths in $2010 ; 6.83 \%$ due to other respiratory diseases) equivalent to 12.54 per 100,000 population. ${ }^{3}$ However the total number (absolute or estimated) of asthmatics in Sri Lanka is not known. If the proportion affected is the same as in India, then simple adjustment for the different population sizes would yield 360,000 adults with asthma in Sri Lanka. ${ }^{29}$

One of the precipitating factors for severe asthma is Aspergillus sensitization, including ABPA. ${ }^{29}$ Five outpatient asthma referral cohorts in South Africa, Ireland, New Zealand, Saudi Arabia and China have estimated the prevalence of ABPA in adults as $2.5 \%$ (range $0.72 \%$ to $3.5 \%) .{ }^{29}$ A hospital-based study in Sri Lanka in 1991 assessed the prevalence of skin test sensitivity to both A. fumigatus and A.niger in Sri-Lankan asthmatics. ${ }^{30}$ Of 134 patients thoroughly evaluated, 38 had severe asthma and 96 had mild or moderate asthma. Eight patients $(6 \%)$ had evidence of ABPA. If we use the international mean rate of ABPA $(2.5 \%)$ and apply it to the estimated total adult asthma population, we would anticipate 9,000 ABPA cases in Sri Lanka, but if we apply the 6\% rate this number would rise to 21,600. Globally, of the 193 million adults with active asthma worldwide, 4,837,000 (range 1,354,000-6,772,000) are estimated to develop ABPA. In South East Asia (WHO region) the estimate was $720,400 .^{29}$

An estimate of severe asthma with fungal sensitization (SAFS) is more difficult because of differing definitions of severe asthma and uncertainty about how generalizable secondary care data are to the whole population. SAFS is diagnosed by the combination of severe asthma, exclusion of ABPA and sensitization to any fungus by skin prick test or specific IgE. SAFS is responsive to oral azole therapy. ${ }^{31}$ In Sri Lanka Aspergillus hypersensitivity is common in asthmatics. Thirty seven percent $(37 \%)$ of patients had skin test reactivity to either or both A. fumigatus and A. niger ( $\mathrm{n}=5$ only A. niger) ${ }^{30}$, which is consistent with other cohorts. ${ }^{32}$ On the assumption that $10 \%$ of the estimated 360,000 adult asthmatics in Sri Lanka have severe asthma, 37\% of whom are sensitized to fungi, an estimate of 13,654 patients with SAFS could be made in Sri Lanka. Global estimate of ABPA is 4.8 million and SAFS is 3.515 million respectively. ${ }^{16}$ 
A 10 year study (1978-1987) detected 106 patients from 462 patients with superficial fungal infections and among them 3.4\% were tinea capitis. The most vulnerable age group was 6-10 years. Dermatophytes accounted for $91 \%$ of the tinea capitis infections. Fifty percent of the lesions were inflammatory (kerion) and the author attributed this to the responsible geophilic and the zoophilic species of dermatophytes. ${ }^{33}$ A household survey based on health interviews and clinical examination was conducted over a period of 4 months in a sub-urban area of Sri Lanka in the late 1990s. In 418 households with1806 residents, the prevalence of skin diseases was $47.6 \%$. Fungal infections, followed by dermatitis were found to be the commonest problems. ${ }^{34}$ Based on unpublished data from the Department of Mycology, Medical Research Institute, Sri Lanka, we estimated 50 fungal hair infections among Sri Lankans annually. This low number can be attributed to Sri Lankan traditional custom of having frequent head baths with soap and water (especially among children) and oiling of scalp. The global disease burden is estimated as 200,000,000 annually. ${ }^{23}$ Another reason could be the lack of proper system of data collection on patients treated by general practitioners and hospital out-patient departments.

Estimation of the number of patients with fungal keratitis in Sri Lanka has never been done. The disease is not rare however, but under suspected and frequently not formally diagnosed. From 2004-2013 the Department of Mycology at Medical Research Institute had tested 1069 (corneal buttons 1058 and corneal scraping samples 11). Among them, 432 samples $(40.41 \%)$ were positive for fungal elements, in direct microscopy. Among the culture positive 407 samples, 402 grew moulds. Majority of them were Fusarium species $(54.54 \%)$ followed by Aspergillus flavus (26.28\%) and Aspergillus fumigatus $(4.66 \%) .{ }^{35,36}$ This provides an estimate of 1277 in Sri Lanka, which is probably a significant underestimate given that infection rates in India and Nepal are 113 and 799 per 100,000 respectively. We assume that the combination of ignorance and under diagnosis especially in the rural population is a likely reason for such low rates. The estimated global fungal eye infection caseload is $1,000,000$ annually.$^{23}$

The proportion of vulvo-vaginal candidiasis (VVC) among women attending the Central STD clinic (Colombo) in Sri Lanka for the first time is $8.33 \% .{ }^{37}$ During this study only one patient was found with recurrent VVC. Studies done at central STD clinic and gynaecology clinics may under represent the problem as social stigma may result in more women seeking medical advice for VVC from general practitioners or buying antifungal preparations over the counter. Due to this, actual figures are unavailable. So based on data from other countries, an estimation of 25,750 women with recurrent VVC was derived (5\% of adult women premenopause). The global disease burden for recurrent VVC is estimated as 137 million. ${ }^{16}$

\section{Rhinosporidiosis}

Sri Lanka has the highest worldwide prevalence of human rhinosporidiosis on a unitpopulation basis. ${ }^{38}$ Rhinosporidiosis is hyper-endemic in India and Sri Lanka. ${ }^{15}$ Though it is hyper-endemic, no recent data is available on the caseload.

\section{Mucormycosis}

This is not an uncommon condition in Sri Lanka. But incidence / prevalence data is not available. We estimated the annual incidence as 41 cases based on 2 per million. 


\section{Pneumocystis}

Since there are no data available for Sri Lanka, we could not estimate the disease burden. Diagnostics for Pneumocystis are in their infancy in Sri Lanka. The estimated global burden for pneumocystis infections is $>200,000$ among the HIV/AIDS patients and $>100,000$ among the immunocompromised and cause 80,000 deaths annually. ${ }^{23} \sim 15 \%$ of the patients with AIDS die and this rate is $\sim 50 \%$ in non-AIDS patients. ${ }^{23}$

\section{Total}

The global estimated number of deaths following fungal diseases is $>1,350,000$ annually. ${ }^{39}$ We estimated the annual fungal disease burden in Sri Lanka as 57,009. But the number of deaths could not be estimated as mortality data was not available.

\section{Conclusion}

The burden of fungal disease in Sri Lanka is substantial, especially respiratory fungal problems. It is essential to diagnose these fungal diseases accurately and rapidly. Good diagnostics are required to more accurately determine the epidemiology of these diseases and assess whether any are emerging diseases in Sri Lanka. To prevent the devastating consequences of these diseases there should be good treatment facilities. Both government and private health institutions should take responsibility for improving diagnostics and treatment. Health personnel should also become better informed and skilled in handling these diseases. More precise data from the whole country is needed for healthcare policy making.

\section{References}

1. Slavin MA, Chakrabarti A. Opportunistic fungal infections in the Asia-Pacific region. Medical Mycology 2012; 50:18-25 doi: 10.3109/13693786.2011.602989

2. Sri Lanka. https://en.wikipedia.org/wiki/Sri_Lanka

3. Health Profile-Sri Lanka- World Life Expectancy. http://www.worldlifeexpectancy.com/country-health-profile/sri-lanka

4. Sri Lanka GDP growth rate. http://www.tradingeconomics.com/sri-lanka/gdp-growth

5. World population ageing 1950-2050 http://www.un.org/esa/population/publications/worldageing19502050/pdf/187srila.pdf

6. Health statistics and information systems. Definition of an older or elderly person WHO http://www.who.int/healthinfo/survey/ageingdefnolder/en/

7. Hospitals and bed strength in Sri Lanka by districts-government sector 2010. http://203.94.76.60/nihs/BEDS/Beds2010/Beds2010.pdf

8. Fernando J, Wickramaratne CP, Dissanayake R, et al. Studying current status of intensive care units in Sri Lanka. Int J Crit Illn Inj Sci 2012; 2:11-16 doi: http://dx.doi.org/10.4103/2229-5151.94884

9. HIV /AIDS in Sri Lanka - World Bank. http://www.worldbank.org/en/news/feature/2012/07/10/hiv-aids-sri-lanka

10. Denning DW, Pleuvry A, Cole DC. Global burden of chronic pulmonary aspergillosis as a sequel to pulmonary tuberculosis. Bull World Health Organ. 2011; 1:89(12); 864-72 doi: 10.2471/BLT.11.089441

11. Jayasekera PI, Perera PD. A ten year retrospective study to evaluate the species of candida in blood cultures received at Department of Mycology, Medical Research Institute Annual Academic Sessions of Sri Lanka College of Microbiologists, 2011; 9:27 
12. Bufford JD, Gern JE, Denning DW, et al. The link between fungi and severe asthma: a summary of the evidence. Eur Respir J 2006; 26:309-18

13. Sobel JD Vulvovaginal candidosis. Lancet 2007; 369 (9577):1961-71

doi: http://dx.doi.org/10.1016/S0140-6736(07)60917-9

14. Lam DS, Houang E, Fan DS, Hong Kong Microbial Keratitis Study Group. Incidence and risk factors for microbial keratitis in Hong Kong: comparison with Europe and North America. Eye (Lond). 2002; 16(5):608-18 doi: 10.1038/sj.eye.6700151

15. Kaluarachchi K, Sumathipala S, Eriyagama N, et al The identification of the natural habitat of Rhinosporidium seeberi with $R$. seeberi-specific in situ hybridization probes J Infect Dis Antimicrob Agents 2008; 2008:25-32

16. Global burden of human fungal infections http://life-worldwide.org/media-centre/article/gaffi-president-delivers-keynote-speech-inspeech-in-montreal-on-global-burden-of-hum

17. Patabendige CGUA, Dimantha, GHG, Jayaratna TS, Perera PD Nosocomial candidaemia at the National Cancer Institute of Sri Lanka (NCISL): A three year prospective study. International Journal of Infectious Diseases 2010; 14 (Sup 2):S44-S45

18. Census of Population \& Housing 2011 http://www.statistics.gov.lk/PopHouSat/CPH2011/index.php?fileName=pop41\&gp=Activities $\& t p l=3$

19. STD quarterly report 2011 http://www.aidscontrol.gov.lk/web/images/pdf/quarterly_reports/quarter4_2011.pdf

20. National STD/AIDS control programme Sri Lanka http://www.aidscontrol.gov.lk/web/index.php/statistics/hiv

21. STD quarterly report 2010 http://www.aidscontrol.gov.lk/web/images/pdf/quarterly_reports/quarter4_2010.

22. World Health Statistics http://www.who.int/whosis/whostat/EN_WHS2011_Full.pdf

23. Brown G.D., Denning D.W., Gow N.A.R. et al., Hidden Killers: Human fungal infections. Sci Transl Med 2012; 4:165 rv13 doi: 10.1126/scitranslmed.3004404

24. TB/HIV in the South-East Asia Region. Status report, December 2011 http://www.searo.who.int/entity/hiv/documents/hiv-aids_in_south-east_asia.pdf

25. Tuberculosis profile 2010. WHO report https://extranet.who.int/sree/Reports

26. Chakrabarti A, Chatterjee SS, Das A, Shivaprakash MR. Invasive aspergillosis in developing countries. Medical Mycology 2011; 49 (Suppl. 1):S35-S47 doi: 10.3109/13693786.2010.505206

27. Montravers P, Mira JP, Gangneux JP, et al. A multicentre study of antifungal strategies and outcome of candida spp. peritonitis in intensive-care units. Clin Microbiol Infect. 2011; 17(7):1061-7.25.25. doi: 10.1111/j.1469-0691.2010.03360.x

28. Sri Lanka Health at a glance 2008, Volume 1. http://www.statistics.gov.lk/Newsletters/Health\%20 Bulletin (Medical\%20Stat).pdf

29. Denning DW, Pleuvry A, Cole DC.et al Global burden of ABPA in adults with asthma and its complication chronic pulmonary aspergillosis. Med Mycol. 2013; 51(4):361-70 doi: 10.3109/13693786.2012.738312

30. Attapattu MC. Allergic Broncho Pulmonary Aspergillosis among asthmatics. Ceylon Med J. 1991; 36: 45-51

31. Denning DW, O'Driscoll BR, Powell G, Randomized controlled trial of oral antifungal treatment for severe asthma with fungal sensitization: The Fungal Asthma Sensitization Trial (FAST) study. Am J Respir Crit Care Med. 2009 1; 179(1):11-8 doi: 10.1164/rccm.200805-737OC

32. Agarwal R. Allergic broncho pulmonary aspergillosis. Chest 2009; 135(3): 805-26 
doi: $10.1378 /$ chest.08-2586

33. Attapattu MC. A study of tinea capitis in Sri Lanka. Journal of Medical and Veterinary Mycology 1989; 27:27-32 No doi

34. Perera A, Atukorale DN, Sivayogan S, et al. Prevalence of skin diseases in sub-urban Sri Lanka. Ceylon Med J. 2000 Sep; 45(3):123-8 No doi

35. Fungal Keratitis Sri Lankan picture. Jayasekera PI, Kudavidanage S, Perera PD. Annual Scientific Sessions of the Sri Lanka College of Microbiologists 2014; 12:23

36. Aetiological agents of fungal keratitis in Sri Lanka. PI Jayasekera, S Kudavidanage, PD Perera. Third Annual Conference and Scientific Sessions of Sri Lankan Society for Microbiology (SSM) - 2014; pp 32

37. Jayasekera, PI, Perera PD, Samarakoon S. Vulvovaginal candidiasis among female patients the Central STD Clinic Colombo, for the first time Annual academic sessions of Sri Lanka College of Microbiologists.2010; 8:22.

38. Sudasinghe T, Rajapakse RP, Perera NA et al,. The regional sero-epidemiology of attending rhinosporidiosis in Sri Lankan humans and animals Acta Trop. 2011; 120 (1-2):72-81 doi: 10.1016/j.actatropica.2011.06.016

39. Fungal Disease Frequency. http://www.gaffi.org/why/fungal-disease-frequency/ 